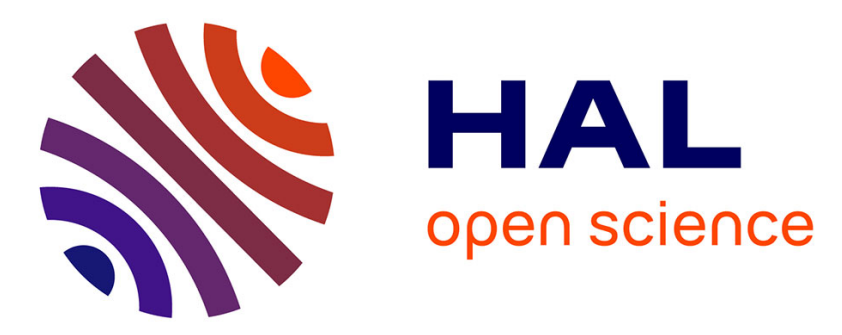

\title{
Biphasic model of lung deformations for Video-Assisted Thoracoscopic Surgery (VATS)
}

\author{
Pablo Alvarez, Saramati Narasimhan, Simon Rouzé, Jean-Louis Dillenseger, \\ Yohan Payan, Michael I Miga, Matthieu Chabanas
}

\section{- To cite this version:}

Pablo Alvarez, Saramati Narasimhan, Simon Rouzé, Jean-Louis Dillenseger, Yohan Payan, et al.. Biphasic model of lung deformations for Video-Assisted Thoracoscopic Surgery (VATS). IEEE International Symposium on Biomedical Imaging (ISBI), Apr 2019, Venice, Italy. inserm-02121765

\section{HAL Id: inserm-02121765 https://www.hal.inserm.fr/inserm-02121765}

Submitted on 6 May 2019

HAL is a multi-disciplinary open access archive for the deposit and dissemination of scientific research documents, whether they are published or not. The documents may come from teaching and research institutions in France or abroad, or from public or private research centers.
L'archive ouverte pluridisciplinaire HAL, est destinée au dépôt et à la diffusion de documents scientifiques de niveau recherche, publiés ou non, émanant des établissements d'enseignement et de recherche français ou étrangers, des laboratoires publics ou privés. 


\title{
BIPHASIC MODEL OF LUNG DEFORMATIONS FOR VIDEO-ASSISTED THORACOSCOPIC SURGERY (VATS)
}

\author{
Pablo Alvarez ${ }^{a, b, e}$, Saramati Narasimhan ${ }^{d, e}$, Simon Rouzé ${ }^{a, c}$, \\ Jean-Louis Dillenseger ${ }^{a}$, Yohan Payan ${ }^{b}$, Michael I. Miga ${ }^{d, e}$, Matthieu Chabanas ${ }^{b, e}$ \\ ${ }^{a}$ Univ. Rennes 1, Inserm, LTSI - UMR 1099, F-35000 Rennes, France \\ ${ }^{b}$ Univ. Grenoble Alpes, CNRS, Grenoble INP, TIMC-IMAG, F-38000 Grenoble, France \\ ${ }^{c}$ CHU Rennes, Department of Cardio-Thoracic and Vascular Surgery, F-35000 Rennes, France \\ ${ }^{d}$ Department of Biomedical Engineering, Vanderbilt University, Nashville, USA \\ ${ }^{e}$ Vanderbilt Institute for Surgery and Engineering, Vanderbilt University, Nashville, USA
}

\begin{abstract}
Intraoperative localization of small, low-density or deep lung nodules during Video-Assisted Thoracoscopic Surgery (VATS) is a challenging task. Localization techniques used in current practice require an additional preoperative procedure that adds complexity to the intervention and might yield to clinical complications. Therefore, clinical practice may benefit from alternative, intraoperative localization methods. We propose a nonrigid registration approach for nodule localization. Our method is based on a biomechanical model of the lung, where the lung parenchyma is represented as a biphasic medium. Preliminary results are promising, with target registration errors reduced from $28.39 \mathrm{~mm}$ to $9.86 \mathrm{~mm}$ in median, and to $3.68 \mathrm{~mm}$ for the nodule in particular.
\end{abstract}

Index Terms - Nonrigid Image Registration, Biomechanical Model, Lung, Video-Assisted Thoracoscopic Surgery

\section{INTRODUCTION}

Surgical resection of lung nodules via Video-Assisted Thoracoscopic Surgery (VATS) is one of the treatments available for early stage lung cancer. In comparison to open thoracotomy, this minimally invasive procedure reduces the length of hospitalization and minimizes post-operative complications [1]. However, at the beginning of the procedure, the insertion of surgical ports and the artificial ventilation applied only on the contralateral lung, allow air to flow into the intrapleural space. This abnormal air inflow, known as a pneumothorax, induces a collapse of the lung towards the hilum, and, therefore large anatomical deformations. As a result, the intraoperative localization of small, deep or low-density nodules becomes considerably difficult [2].

In current practice, localization techniques rely on preoperative procedures for nodule marking, generally involving the placement of hookwires or dyes under CT fluoroscopy [3]. However, these procedures not only increase the cost and complexity of care, but also occasionally lead to serious complications [4]. As an alternative, several groups have recently proposed localization methods based on intraoperative imaging only, for example, Cone Beam CT (CBCT) [5] or Ultrasound [6]. While promising, these methods rely entirely on nodule visibility in the images, which may be insufficient. For instance, the fuzzy borders and low-density of ground glass opacity nodules could make them indistinguishable, especially considering the increased density of the collapsed lung. A potential solution to address this limitation could be nonrigid image registration, to transfer information from the preoperative $\mathrm{CT}$ to the deformed intraoperative configuration.

Many studies have addressed the registration of lung images, with methods based on image intensity [7], biomechanical models [8, 9], or a combination of both [10]. The purpose of these methods is to compensate for the lung deformations during normal breathing, mainly for conformational radiation therapy. However, lung deformations are considerably larger during VATS [11], which increases the difficulty of the registration problem. To the best of our knowledge, only one study has proposed the use of nonrigid image registration in the context of VATS using CBCT images of a pig lung [12].

In this preliminary work, we propose to compensate for lung deformation using a nonrigid image registration approach guided by a biomechanical model of the lung. Using clinical data with deformations consistent to those of VATS, we modeled the lung as a biphasic medium and estimated its deformation via finite element simulation. The main objective was to evaluate the capacity of the model to estimate lung deformations during VATS. We intend to use this approach as an intraoperative nodule localization strategy for VATS.

\section{MATERIALS AND METHODS}

\subsection{Data}

This retrospective study was conducted on a single clinical case. After a CT image was acquired, the patient had a nee- 


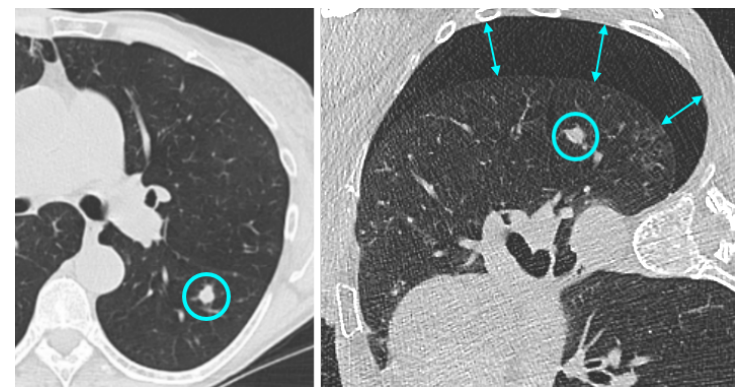

Fig. 1. Left lung containing a solid, solitary nodule, indicated with a circle. Left: Axial cut of the CT image with the patient in supine position. Right: Axial cut of the LDCT image after pneumothorax with the patient in lateral decubitus position. The pneumothorax is indicated by arrows.

dle biopsy of a solitary nodule located in his left lung. During this procedure, the patient developed a pneumothorax that was later detected with a post-interventional Low Dose CT (LDCT) image. Although this clinical case is not a VATS intervention, the resulting lung deformation is very similar. Specifically, there is a change of patient pose from supine during the CT imaging to lateral decubitus during the LDCT imaging, as well as a pneumothorax (Fig. 1).

Several structures were manually segmented from these images: the airway tree, lung parenchyma and nodule from the CT image; and the deflated lung parenchyma, thoracic cage, and nodule from the LDCT image.

\subsection{Finite element model}

In this work, the biphasic model proposed in [13] was used to represent the lung. This model is based on Biot's theory of soil consolidation [14], and has been previously used for brain shift compensation [15]. The governing equations are:

$$
\begin{aligned}
\nabla \cdot(G \nabla \mathbf{u})+\nabla \frac{G}{(1-2 \nu)}(\nabla \cdot \mathbf{u})-\nabla p & =-\rho \mathbf{g} \\
-\nabla \cdot(k \nabla p)+k_{c}\left(p-p_{c}\right) & =0
\end{aligned}
$$

where $\mathbf{u}$ is the displacement vector, $p$ is the interstitial pressure, $\nu$ is the Poisson's ratio, $G$ is the shear modulus defined by $\frac{E}{2(1+\nu)}$ with $E$ as Young's modulus, $\rho$ is a density measure defined by $\left(\rho_{f}-\rho_{s}\right)$ with $\rho_{f}$ and $\rho_{s}$ as the density of the fluid and solid phases respectively, $\mathrm{g}$ is the gravitational unit vector, $k_{c}$ is the bronchi permeability, $p_{c}$ is the internal bronchi pressure and $k$ the hydraulic conductivity. In addition, we should note that the interchange of air between the lung parenchyma and small bronchi has been approximated by lumping these exchange-effects using the organ-wide distributed term $k_{c}\left(p-p_{c}\right)$. It allows the simulation of air evacuation from small-scale lower bronchi structures distributed throughout the lung parenchyma, therefore producing local volume changes.

\subsection{Geometry reconstruction}

Using CGAL library (www.cgal.org), two finite element meshes of the lung with four-node-tetrahedral elements were generated (Fig. 2). The first mesh $\left(L_{c t}\right)$ was generated from the lung parenchyma segmented in the CT image. The second mesh $\left(L_{l d c t}\right)$ was generated from the the thoracic cage segmented in the LDCT image. For the latter, the underlying assumption is that the original shape of the lung (i.e. before pneumothorax) matches the shape of the thoracic cage.

\subsection{Material properties}

As shown in previous studies, the lung parenchyma is highly heterogeneous [8]. The Young's modulus $E$, for instance, is considered to be lowest near the diaphragm and highest near the hilum. Similarly, we have made the approximation that the hydraulic conductivity $k$ decreases significantly with the distance from the main airways. The natural anatomic structure of the lung with its decreasing bronchi diameter scale would result in a more restricted exchange, or a lower hydraulic conductivity.
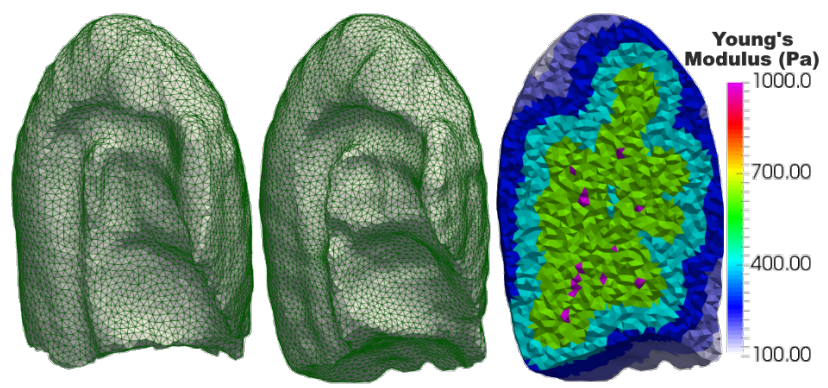

Fig. 2. Left: Tetrahedral mesh of the lung parenchyma from the CT image $\left(L_{c t}\right)$. Middle: Tetrahedral mesh of the thoracic cage from the LDCT image $\left(L_{l d c t}\right)$. Right: Example of mesh stratification on $L_{l d c t}$ and the corresponding values of Young's modulus $E\left(E_{\min }=0.1 \mathrm{kPa}\right.$ and $\left.E_{\max }=1 \mathrm{kPa}\right)$.

Similar to the hydraulic conductivity, we have adopted a distance-to-airway dependence with Young's modulus. As the arrangement of bronchi cartilage rings and subsequent structural elements is present, an approximation of tissue heterogeneity is proposed to decrease Young's modulus with increasing distance from these structures. We have used distance ranges of approximately $15 \mathrm{~mm}(0-15 \mathrm{~mm}, 15$ $30 \mathrm{~mm}$, etc.) to vary the Young's modulus as an exponential function of this distance (Fig. 2 right). This relationship is represented by:

$$
E(d)=\alpha e^{-\gamma d}+\beta
$$

where $d$ is the distance to the airway tree and $\gamma$ is the decay constant. Constants $\alpha$ and $\beta$ are computed based on fit values of $E_{\min }$ and $E_{\max }$ such that $E\left(d_{\min }\right)=E_{\max }$ 
and $E\left(d_{\max }\right)=E_{\min }$. An analogous exponential function was used for the hydraulic conductivity $k$ values, based on fit values of $k_{\min }$ and $k_{\max }$.

\subsection{Initial alignment}

Before nonrigid deformation, registration to a common reference frame was performed. We aligned the spine from the CT and LDCT images using rigid body registration guided by Mutual Information. This procedure was performed with the help of the multi-resolution, image-based registration techniques implemented in Elastix toolbox [16].

\subsection{Deformation compensation strategy}

The deformation experienced by the lung from the CT configuration to the LDCT configuration might be understood as a combination of three interdependent events: (1) a change in the direction of gravity and its effects on internal structures; (2) a change in patient pose that affects the position and shape of the lung; and (3) the deflation of the lung as a result of pneumothorax. The true deformation is an intricate combination of the individual deformations resulting from each one of these events. In this preliminary study, we explored the feasibility of a linear biomechanical model to estimate these lung deformations. The three deformations were estimated independently before being superposed to produce the final post-pneumothorax registered CT image.

Deformations (1) and (2) dealt with the estimation of the pre-pneumothorax anatomy of the lung in the LDCT image configuration. Deformation (3) used the previously estimated anatomy to calculate the deformation associated with only the pneumothorax. It is worth mentioning that our approach does not employ a moving grid from stage to stage so geometric nonlinearities are neglected. We should also note that no hereditary approach accounting for strain history has been implemented. Rather, in this preliminary work, we have estimated each condition from a single mesh pose and deformation was a simple linear combination of deformation modes.

\subsubsection{Gravity compensation}

The direction of gravity with respect to the patient is different in the CT and LDCT configurations. We compensated for this difference by first estimating a gravity-free state of the lung in the CT configuration, and then recomputing the effects of gravity in the LDCT configuration. For the gravity-free state, the deformation was computed on $L_{c t}$ by applying a body force with the magnitude of gravity, but in the opposite direction, i.e. the right hand side of Eq. (1) becomes positive.

Tissue parameters were assigned as described in section 2.4. using empirically-found values within the range suggested in [8]. The fluid medium was disregarded during the simulation to compute this deformation. In addition, nodes on the boundaries of the parenchyma were allowed to slide along the surface, while assuming no friction between the visceral and parietal pleurae.

\subsubsection{Change of pose compensation}

Notable deformation is introduced in the thorax after the change of patient pose [11]. We used a surface matching strategy to compensate for this deformation, where displacements were imposed on surface nodes of $L_{c t}$ to match the surface of $L_{l d c t}$.

First, the borders of the thoracic cage of the CT and LDCT images were matched using a BSpline-based Free-Form Deformation (FFD) registration algorithm guided by Mutual Information, as implemented in Elastix toolbox [16]. From this, we obtained a displacement map describing the deformation necessary to align the surface of $L_{c t}$ to that of $L_{l d c t}$. Then, we imposed displacements on each node of the surface of $L_{c t}$, according to the displacement map computed previously. Material properties were designated as in the previous step.

\subsubsection{Pneumothorax compensation}

We modeled the pneumothorax as being caused by differences in pressure between the surface and the interior of the parenchyma. The fluid mass conservation law associated with equation (2) allows for the evacuation of the fluid phase (air) via the bronchi and the subsequent volumetric contraction of tissue.A positive pressure $\left(5 \mathrm{~cm} \mathrm{H}_{2} \mathrm{O}\right)$ was assigned to the surface nodes of $L_{l d c t}$, while remaining nodes had a noflux boundary condition. Bronchi permeability was addressed with $k_{c}$ and $p_{c}$, with pressure $p_{c}$ equals to zero inside the airways. Additionally, the deflation of the lung is constrained by the boundaries of the thoracic cage. Therefore, we assigned slip conditions to the nodes in contact with the thoracic cage, as visualized in the LDCT image.

Since, to the best of our knowledge, the material properties used in the governing equations have never been established for the pneumothorax, we approximated these values using the trust-region nonlinear optimization method implemented in MATLAB. The 7 parameters being fit for in this inverse model were: $E_{\max }, E_{\min }, k_{\max }, k_{\min }, \gamma, \rho$ and $k_{c}$. We iteratively minimized the difference between our modelestimated deformation and the true deformation measured in the LDCT image. More specifically, we used a measure of surface-to-surface proximity between the current (model) and target (LDCT) deformations as the optimality criteria. That is, we minimized the objective function $\Omega$ defined as:

$$
\Omega=\frac{1}{N} \sum_{i=1}^{N}\left(\mathbf{x}_{d, i}-\mathbf{x}_{t, i}, \mathbf{n}_{t, i}\right)^{2}
$$

where $N$ is the number of deformed surface points, $\mathbf{x}_{d, i}$ is an indexed deformed-surface point, $\mathbf{x}_{t, i}$ is the corresponding closest target-surface point, $\mathbf{n}_{t, i}$ is the normal of the target surface on $\mathbf{x}_{t, i}$, and $(\cdot, \cdot)$ is the inner product operator. The 
objective function $\Omega$ is a variant of the point-to-point distance that takes into account the local shape of the target surface. We clarify that $\mathbf{x}_{d}$ comes from the surface of $L_{l d c t}$ after simulation, and $\mathbf{x}_{t}$ comes from the surface of the deflated lung, in the LDCT image.

\section{Deformation superposition}

The computed deformations were superposed to obtain the post-pneumothorax registered CT image. First, deformations (1) and (2) were added on every node of $L_{c t}$. The resulting deformation field was used to warp the CT image into the pre-pneumothorax, LDCT configuration. Then, this synthetic image was warped again using deformation (3) on $L_{l d c t}$ to produce the final post-pneumothorax registered CT image.

\section{EXPERIMENTAL RESULTS}

Our approach was validated using Target Registration Errors (TRE) on a set of 22 paired anatomical landmarks. Twentyone of those landmarks were localized in clearly identifiable airway and vessel bifurcations, covering a large portion of the lung volume. Finally, an additional landmark was defined in the center of the lung nodule.

Figure 3 collects the resulting TREs after three different levels of deformation compensation. First, we applied the rigid initial alignment only, which provides an order of magnitude for the deformation. Then, we applied the pneumothorax compensation step only, to evaluate the relevance of accounting for the gravity and the change of pose. Finally, we evaluated our whole deformation compensation strategy.

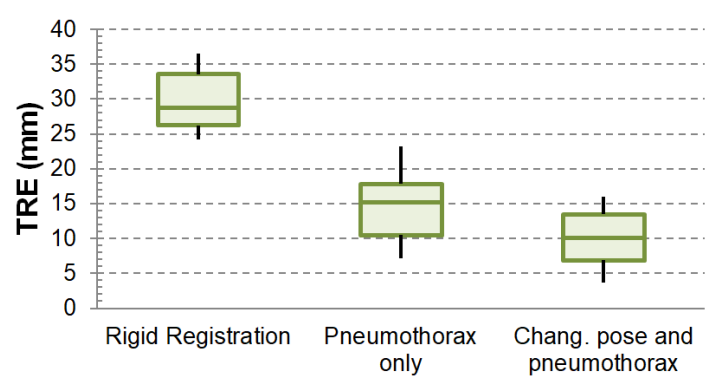

Fig. 3. TREs after three levels of deformation compensation.

A graphical representation of the spatial distribution of post-deformation anatomical landmarks is depicted in Fig. 4. Each landmark is displayed with a color indicating final TRE. In particular, the landmark positioned in the nodule had a TRE of $28.39 \mathrm{~mm}$ after initial alignment, $8.06 \mathrm{~mm}$ after correcting for the pneumothorax only, and $3.64 \mathrm{~mm}$ after applying the whole deformation compensation strategy.

Finally, the parameters found in the optimization procedure were as follows: $E_{\max }=0.94 \mathrm{kPa}, E_{\min }=0.14 \mathrm{kPa}$, $k_{\text {max }}=1.7 \times 10^{-12} \mathrm{~m}^{3} \mathrm{~s} / \mathrm{kg}, k_{\text {min }}=1.5 \times 10^{-13} \mathrm{~m}^{3} \mathrm{~s} / \mathrm{kg}$, $\gamma=0.28, \rho=165.96 \mathrm{~kg} / \mathrm{m}^{3}$ and $k_{c}=2.51 \times 10^{-9} \mathrm{~Pa} / \mathrm{s}$.
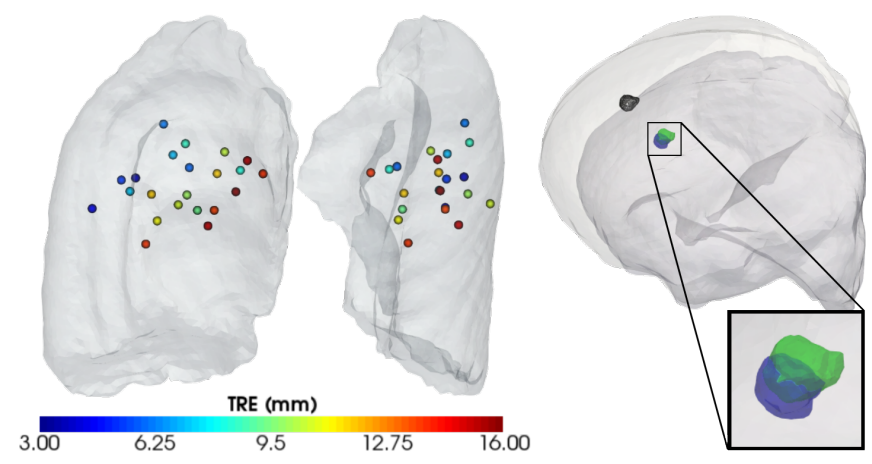

Fig. 4. Left: Spatial distribution of post-deformation landmarks represented with a color code indicating TREs. Right: $3 \mathrm{D}$ reconstructions of the ground truth tumor (in blue), deformation compensated tumor (in green) and rigidly transformed tumor (in black). The surfaces represent $L_{l d c t}$ before and after simulation of pneumothorax.

\section{DISCUSSION AND CONCLUSION}

Preliminary results suggest that our proposed nonrigid registration approach could compensate for lung deformations occurring during VATS. The reported TREs are promising, considering that the necessary negative margins for nodule resection in VATS are approximately $15 \mathrm{~mm}$ [17]. In addition, we have also shown the importance of accounting for the change of pose of the patient, and gravity. To that end, it is interesting to note that our preliminary linear superposition of the three deformations resulted in improvements in terms of TRE.

As tissue parameters of the lung are patient-dependent, we did not know the property values required to induce the pneumothorax deformation. For this reason, we used optimization as the tool to estimate these tissue parameters, which ultimately dictated the required deformation. The found values were consistent with previous studies [8, 18]. We could then use these values to test the ability of the proposed model to reproduce the lung deformation occurring during VATS.

Future work will include validating our method with more clinical data, to reflect varying degrees of pneumothorax and different nodule locations. Also, the registration approach will have to be adapted for intraoperative CBCT images, by dealing with partial lung anatomy and increased image noise.

\section{Acknowledgments}

This work was supported by the Région Bretagne through its Allocations de Recherche Doctorale (ARED) framework; the French National Research Agency (ANR) through the frameworks Investissements d'Avenir Labex CAMI (ANR11-LABX-0004) and Infrastructure d'Avenir en Biologie et Santé (ANR-11-INBS-0006); and the National Institutes of Health - NINDS grant R01NS049251. 


\section{REFERENCES}

[1] P.-E. Falcoz, M. Puyraveau, P.-A. Thomas, H. Decaluwe, M. Hürtgen, R. H. Petersen, H. Hansen, and A. Brunelli, "Video-assisted thoracoscopic surgery versus open lobectomy for primary non-small-cell lung cancer: a propensity-matched analysis of outcome from the European Society of Thoracic Surgeon database," European Journal of Cardio-Thoracic Surgery, vol. 49, no. 2, pp. 602-609, Feb. 2016.

[2] M. K. Gould, J. Donington, W. R. Lynch, P. J. Mazzone, D. E. Midthun, D. P. Naidich, and R. S. Wiener, "Evaluation of Individuals With Pulmonary Nodules: When Is It Lung Cancer?," Chest, vol. 143, no. 5, pp. e93Se120S, 2013.

[3] J. Keating and S. Singhal, "Novel Methods of Intraoperative Localization and Margin Assessment of Pulmonary Nodules," Seminars in Thoracic and Cardiovascular Surgery, vol. 28, no. 1, pp. 127-136, 2016.

[4] C. H. Park, K. Han, J. Hur, S. M. Lee, J. W. Lee, S. H. Hwang, J. S. Seo, K. H. Lee, W. Kwon, T. H. Kim, and B. W. Choi, "Comparative Effectiveness and Safety of Preoperative Lung Localization for Pulmonary Nodules," Chest, vol. 151, no. 2, pp. 316-328, Feb. 2017.

[5] S. Rouzé, B. de Latour, E. Flécher, J. Guihaire, M. Castro, R. Corre, P. Haigron, and J.-P. Verhoye, "Small pulmonary nodule localization with cone beam computed tomography during video-assisted thoracic surgery: a feasibility study," Interactive CardioVascular and Thoracic Surgery, vol. 22, no. 6, pp. 705-711, June 2016.

[6] H. Wada, T. Anayama, K. Hirohashi, T. Nakajima, T. Kato, T. K. Waddell, S. Keshavjee, I. Yoshino, and K. Yasufuku, "Thoracoscopic ultrasonography for localization of subcentimetre lung nodules," European Journal of Cardio-Thoracic Surgery, vol. 49, no. 2, pp. 690-697, Feb. 2016.

[7] K. Murphy, B. van Ginneken, J. M. Reinhardt, S. Kabus, and Kai Ding et al., "Evaluation of Registration Methods on Thoracic CT: The EMPIRE10 Challenge," IEEE Transactions on Medical Imaging, vol. 30, no. 11, pp. 1901-1920, Nov. 2011.

[8] B. Seyfi, A. P. Santhanam, and O. J. Ilegbusi, "Effect of gravity on subject-specific human lung deformation," Mathematical and Computer Modelling of Dynamical Systems, vol. 24, no. 1, pp. 87-101, Jan. 2018.

[9] A. Al-Mayah, J. Moseley, M. Velec, S. Hunter, and K. Brock, "Deformable image registration of heterogeneous human lung incorporating the bronchial tree: Deformable image registration of heterogeneous human lung," Medical Physics, vol. 37, no. 9, pp. 4560-4571, Aug. 2010.

[10] L. Han, H. Dong, J. R. McClelland, L. Han, D. J. Hawkes, and Dean C. Barratt, "A hybrid patient-specific biomechanical model based image registration method for the motion estimation of lungs," Medical Image Analysis, vol. 39, pp. 87-100, July 2017.

[11] P. Alvarez, M. Chabanas, S. Rouzé, M. Castro, J.-L. Dillenseger, and Y. Payan, "Lung deformation between preoperative CT and intraoperative CBCT for thoracoscopic surgery: a case study," in Proc. SPIE Medical Imaging 2018: Image-Guided Procedures, Robotic Interventions, and Modeling, 2018, pp. 1-7.

[12] A. Uneri, S. Nithiananthan, S. Schafer, Y. Otake, J. W. Stayman, G. Kleinszig, M.S. Sussman, J.L. Prince, and J.H. Siewerdsen, "Deformable registration of the inflated and deflated lung in cone-beam CT-guided thoracic surgery: Initial investigation of a combined modeland image-driven approach," Medical physics, vol. 40, no. $1,2013$.

[13] M. I. Miga, K. D. Paulsen, J. M. Lemery, S. D. Eisner, A. Hartov, F. E. Kennedy, and D. W. Roberts, "Model-updated image guidance: initial clinical experiences with gravity-induced brain deformation," IEEE Transactions on Medical Imaging, vol. 18, no. 10, pp. 866-874, Oct 1999.

[14] M. A. Biot, "General Theory of Three Dimensional Consolidation," Journal of Applied Physics, vol. 12, no. 2, pp. 155-164, Feb. 1941.

[15] K. Sun, T. S Pheiffer, A. L. Simpson, J. A Weis, R. C. Thompson, and M. I. Miga, "Near real-time computer assisted surgery for brain shift correction using biomechanical models," IEEE J Transl Eng Health Med, vol. 2, no. 2, pp. 2014, 2014.

[16] S. Klein, M. Staring, K. Murphy, M.A. Viergever, and J. Pluim, "elastix: A Toolbox for Intensity-Based Medical Image Registration," IEEE Transactions on Medical Imaging, vol. 29, no. 1, pp. 196-205, Jan. 2010.

[17] K. Mohiuddin, S. Haneuse, T. Sofer, R. Gill, M. T. Jaklitsch, Y. L. Colson, J. Wee, R. Bueno, S. J. Mentzer, D. J. Sugarbaker, and S. J. Swanson, "Relationship between margin distance and local recurrence among patients undergoing wedge resection for small $(\leq 2 \mathrm{~cm})$ non-small cell lung cancer," The Journal of Thoracic and Cardiovascular Surgery, vol. 147, no. 4, pp. 1169-1177, 2014.

[18] P. Dumpuri, R. C. Thompson, B. M. Dawant, A. Cao, and M. I. Miga, "An atlas-based method to compensate for brain shift: Preliminary results," Medical Image Analysis, vol. 11, no. 2, pp. 128-145, Apr. 2007. 